\title{
Outage analysis of full-duplex relaying with co-channel interference
}

\author{
Xiaohui $\mathrm{Hu}^{1, \mathrm{a}}$ and Xianyi Rui ${ }^{2, \mathrm{~b}}$ \\ ${ }^{1}$ School of Electronic and Information Engineering,Soochow University,Suzhou 215006, \\ China \\ ${ }^{2}$ School of Electronic and Information Engineering,Soochow University, Suzhou 215006, \\ China

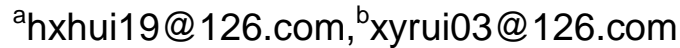

Keywords: full-duplex relaying、 co-channel interference、 decode-and-forward

\begin{abstract}
This paper focuses on the full-duplex relaying with co-channel interference. Some closed-form expressions for outage probability of a decode-and-forward relay system are derived and the independent but not identically distributed Rayleigh fading is assumed. The analytical results are compared with the simulated results and a good agreement is obtained.
\end{abstract}

\section{Introduction}

In recent years cooperative relaying communications has attracted more attention from the wireless communications area thanks to the increase of coverage and throughput without the higher power levels at transmitter end. Most of the existing work on cooperative diversity has mainly concerned about single-cell environments without the co-channel interference [1-2]. On the other hand, because more new demand for wireless communication services continues to increase, frequency reuse has been demonstrated to be a practical technique which can efficiently utilize the radio spectrum. However, as is well known to all, the frequency reuse can cause co-channel interference and it consequently causes severe performance degradation. In [3], performance of a two-hop amplify-and-forward system with multiple interferences at the relay end has been analyzed. The authors of [4], have investigated outage analysis of a two-hop relay system with fixed-gain and multiple Rayleigh interferences at the both relay and destination. Recently, in [5] outage analysis of a multiuser relay system employing opportunistic scheduling in the presence of interference has been investigated. However, the work mentioned above is founded on the hypothesis of the relay operateing in half-duplex mode. In practical systems, full-duplex relaying [6-7] can have the better spectral efficiency performance than half-duplex relaying.

In this paper, the closed-form expression for outage performance is derived for a decode-and-forward (DF) relay system with full-duplex relaying and co-channel interference when the independent but not identically distributed (INID) Rayleigh fading channels are assumed.

\section{System model}

We focus on a two-hop relay system where one source $(S)$ transmits signals to one destination (D) with the aid of a DF relay (R). In our system model, we assume that there is no direct link between nodes $\mathrm{S}$ and $\mathrm{D}$. Half-duplex mode is used in the nodes $\mathrm{S}$ and $\mathrm{D}$, which are equipped with a single antenna, but full-duplex mode is used in the 
node $\mathrm{R}$ with one receive antenna and one transmit antenna. Let $h_{S R}$ denote a complex Gaussian channel between the nodes $\mathrm{S}$ and $\mathrm{R}$ with $E\left[\left|h_{S R}\right|^{2}\right]=\Omega_{S R}$ and zero mean. The expectation operator is with $E[\cdot]$. At the relay, the received signal can be written by

$$
y_{R}=h_{S R} x_{S}+h_{I} x_{R}+n
$$

where $x_{s}$ and $x_{R}$ are the data symbols from the nodes $\mathrm{S}$ and $\mathrm{R}$, respectively. It is assumed that $h_{\mathrm{I}}$ is the self-interference channel between output and input of the relay with $E\left[\left|h_{I}\right|^{2}\right]=\Omega_{I}$. And $n$ is the additive white Gaussian noise terms with zero mean and the variance $E\left[|n|^{2}\right]=\sigma_{n}^{2}$. On the right side of equation in (1), the second item represents the self-interference within node $\mathrm{R}$ [6-7]. So, the signal-to-interference and noise ratio (SINR) in the S-R link can be obtained by

$$
\gamma_{R}=\frac{P_{S}\left|h_{S R}\right|^{2}}{P_{R}\left|h_{I}\right|^{2}+\sigma_{n}^{2}}=\frac{\gamma_{0}\left|h_{S R}\right|^{2}}{\eta_{0}\left|h_{I}\right|^{2}+1}=\frac{\gamma_{0} \lambda_{1}}{\eta_{0} \lambda_{I}+1}
$$

where $P_{S}$ and $P_{R}$ are the transmit power of nodes $\mathrm{S}$ and $\mathrm{R}$ respectively, $\gamma_{0}=P_{S} / \sigma_{n}^{2}$, $\eta_{0}=P_{R} / \sigma_{n}^{2}, \eta_{0}=P_{R} / \sigma_{n}^{2}, \quad \lambda_{1}=\left|h_{S R}\right|^{2}$ and $\lambda_{I}=\left|h_{I}\right|^{2}$.

At node $\mathrm{D}$, the received signal in the presence of a co-channel interferer $x_{i}$, each with an average power $P_{i}$, can be written by

$$
y_{D}=h_{R D} x_{R}+h_{i} x_{i}+n
$$

where $h_{R D}$ is a complex Gaussian channel between R and D with $E\left[\left|h_{R D}\right|^{2}\right]=\Omega_{R D}$ and zero mean, $h_{i}$ is the independent complex Gaussian channel between the a co-channel interferer and D with $E\left[\left|h_{i}\right|^{2}\right]=\Omega_{i}$ and zero mean.

Accordingly, the SINR of the R-D link can be written by

$$
\gamma_{D}=\frac{P_{R}\left|h_{R D}\right|^{2}}{P_{i}\left|h_{i}\right|^{2}+\sigma^{2}}=\frac{\eta_{0} \lambda_{2}}{\eta_{i} \lambda_{i}+1}
$$

where $\eta_{i}=P_{i} / \sigma_{n}^{2}, \quad \lambda_{2}=\left|h_{R D}\right|^{2}$ and $\lambda_{i}=\left|h_{i}\right|^{2}$.

As to the DF scheme, the end-to-end output SINR at the destination (D) may be well approximated under the high SNR condition [8].

$$
\gamma_{e q}=\min \left\{\gamma_{R}, \gamma_{D}\right\}
$$

\section{Outage probability}

In this section, the closed-form expression for outage performance is derived for a DF relay system with full-duplex relaying and co-channel interference.

\subsection{CDF of $\gamma_{R}$}

Unlike the half-duplex relaying scheme, full-duplex relaying introduces self-interference channel between output and input at the relay. For the S-R link, the CDF of $\gamma_{R}$ in (2) can be given by

$$
F_{\gamma_{R}}(\gamma)=\operatorname{Pr}\left\{\frac{\gamma_{0} \lambda_{1}}{\eta_{0} \lambda_{I}+1}<\gamma\right\}=\int_{0}^{\infty} \int_{0}^{\frac{\left(\eta_{0} y+1\right) \gamma}{\gamma_{0}}} f_{\lambda_{1}}(x) f_{\lambda_{I}}(y) d x d y
$$


where $f_{\lambda_{1}}(x)=\alpha_{1} e^{-\alpha_{1} x}$ and $f_{\lambda_{I}}(y)=\alpha_{I} e^{-\alpha_{I} y}$ are probability density function (PDF) of $\lambda_{1}$ and $\lambda_{I}$ with $\alpha_{1}=1 /\left(\gamma_{0} \Omega_{S R}\right)$ and $\alpha_{I}=1 /\left(\eta_{0} \Omega_{I}\right)$. After a series of mathematical manipulations, the expression in closed-form for CDF of $\gamma_{R}$ can be expressed by

$$
F_{\gamma_{R}}(\gamma)=1-\frac{\alpha_{I} \gamma_{0} e^{-\alpha_{1} \gamma / \gamma_{0}}}{\alpha_{1} \eta_{0} \gamma+\alpha_{I} \gamma_{0}}
$$

\subsection{CDF of $\gamma_{D}$}

For the second hop, the CDF of $\gamma_{D}$ in (4) can be given by

$$
F_{\gamma_{D}}(\gamma)=\operatorname{Pr}\left\{\frac{\eta_{0} \lambda_{2}}{\eta_{i} \lambda_{i}+1}<\gamma\right\}=\int_{0}^{\infty} \int_{0}^{\frac{\left(\eta_{i} y+1\right) \gamma}{\eta_{0}}} f_{\lambda_{2}}(x) f_{\gamma_{i}}(y) d x d y
$$

where $f_{\lambda_{2}}(x)=\alpha_{2} e^{-\alpha_{2} x}$ and $f_{\lambda_{i}}(y)=\alpha_{i} e^{-\alpha_{i} y}$ are probability density function (PDF) of $\lambda_{1}$ and $\lambda_{I}$ with $\alpha_{2}=1 /\left(\eta_{0} \Omega_{R D}\right)$ and $\alpha_{i}=1 /\left(\eta_{i} \Omega_{i}\right)$. After a series of mathematical manipulations, the expression in closed-form for CDF of $\gamma_{D}$ can be expressed by

$$
F_{\gamma_{D}}(\gamma)=1-\frac{\alpha_{i} \eta_{0} e^{-\alpha_{2} \gamma / \eta_{0}}}{\alpha_{2} \eta_{i} \gamma+\alpha_{i} \eta_{0}}
$$

\subsection{Outage probability}

Outage probability is a major scale for a wireless communication system and can be defined as a probabilit $P_{\text {out }}\left(C_{\text {th }}\right)$ that the instantaneous mutual information $I=\log _{2}\left(1+\gamma_{e q}\right)$ is less than a target rate $C_{t h}$. Therefore, a simple closed-form expression for outage probability can be given by

$$
P_{\text {out }}\left(C_{\text {th }}\right)=\operatorname{Pr}\left\{I<C_{\text {th }}\right\}=\operatorname{Pr}\left\{\gamma_{\text {eq }}<2^{C_{\text {th }}}-1\right\}=F_{\text {eq }}\left(2^{C_{\text {th }}}-1\right)
$$

where $F_{e q}(\gamma)$ denotes the CDF of $\gamma_{e q}$ in (5) and can be derived by

$$
\begin{aligned}
F_{e q}(\gamma) & =\operatorname{Pr}\left\{\gamma_{e q}=\min \left\{\gamma_{R}, \gamma_{D}\right\}<\gamma\right\} \\
& =1-\left[1-F_{R}(\gamma)\right]\left[1-F_{D}(\gamma)\right]
\end{aligned}
$$

Submitting (7) and (9) into (11), outage probability in (10) can be expressed as

$$
P_{\text {out }}\left(\gamma_{\text {th }}\right)=1-\frac{\alpha_{I} \alpha_{i} \gamma_{0} \eta_{0} e^{-\alpha_{1} \gamma_{\text {th }} / \gamma_{0}-\alpha_{2} \gamma_{\text {th }} / \eta_{0}}}{\left(\alpha_{1} \eta_{0} \gamma_{\text {th }}+\alpha_{I} \gamma_{0}\right)\left(\alpha_{2} \eta_{i} \gamma_{\text {th }}+\alpha_{i} \eta_{0}\right)}
$$

\section{Numerical and simulated results}

In the section, we present some analytical and simulation results to evaluate outage performance of full-duplex relay networks with co-channel interference. 


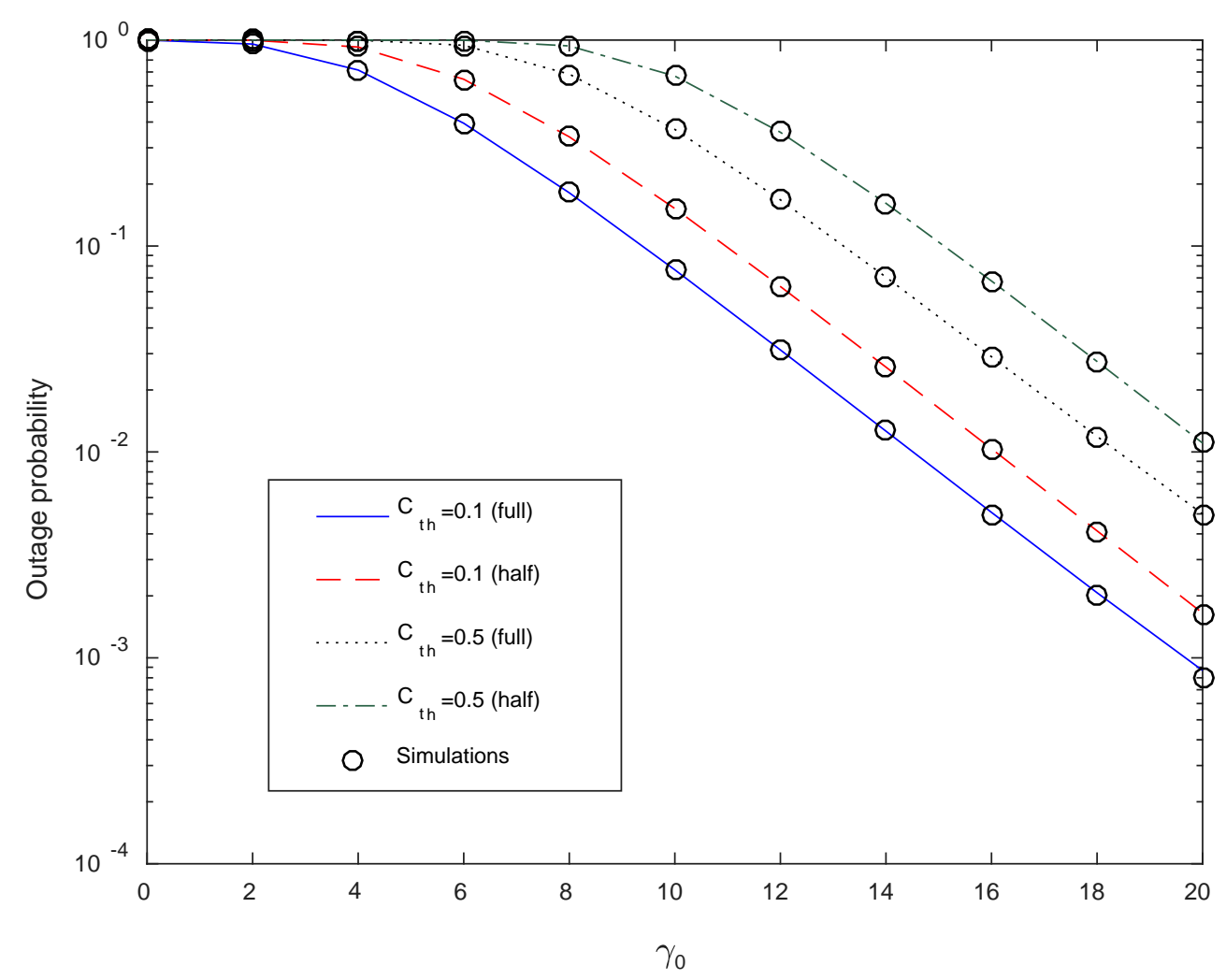

Figure 1. Outage probability vs. $\gamma_{0}$

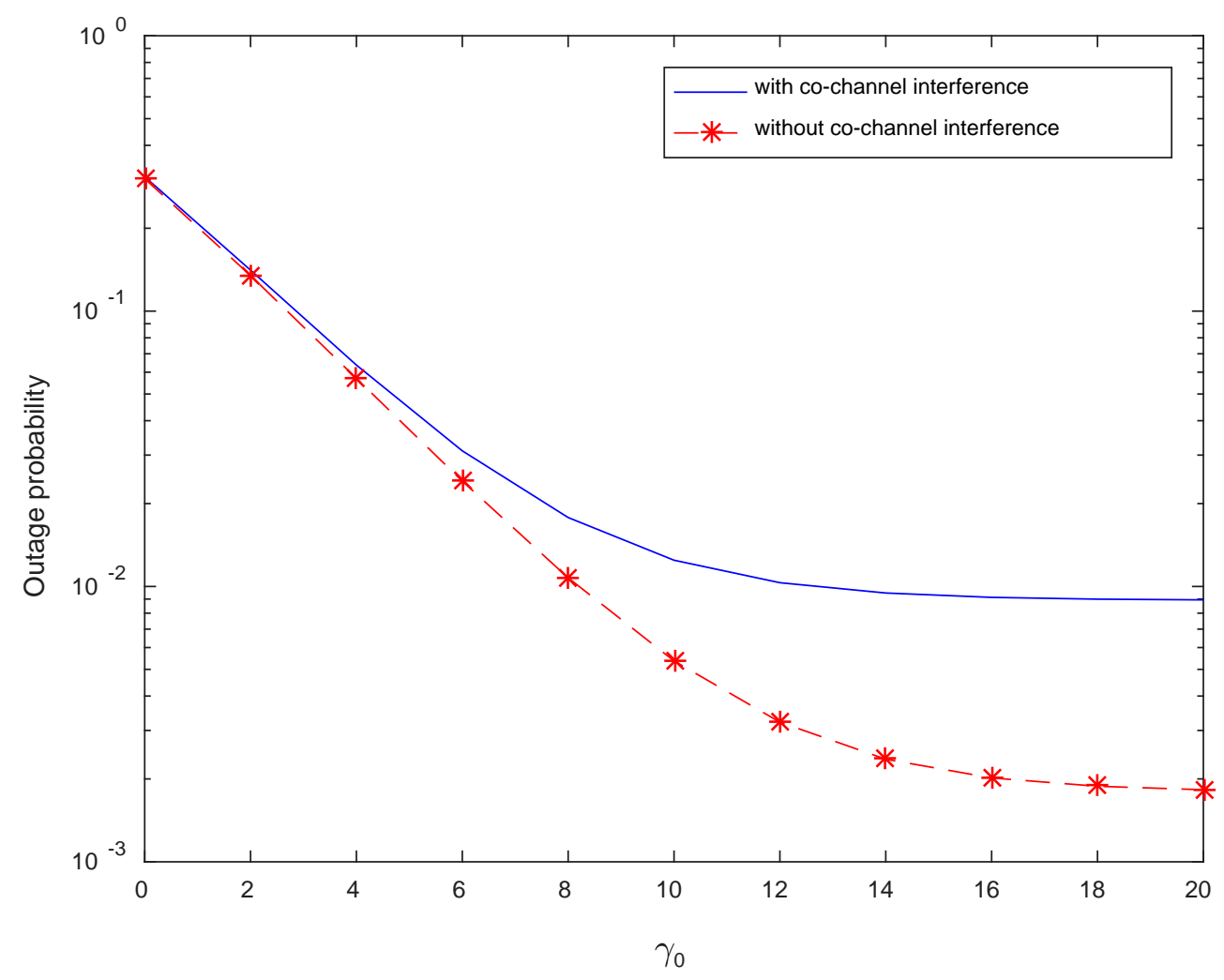

Figure 2. Outage probability with and without co-channel interference 
Fig.1 plots outage probability curves based on (13) when $\Omega_{S R}=\Omega_{R D}=1$ and $\Omega_{I}=0.1$. For simplicity, it is assumed that $\eta_{0}=\gamma_{0} / 10$, and every interference channel parameters $\Omega_{i}=0.1$ and $\eta_{i}=0 d B$. For comparison, we also provide the results of half-duplex relaying. From this figure, we can observe that full-duplex relaying has the better outage performance. In the first figure, the agreement between the simulated and analytical curves validates the accuracy of our analytical formula in (13). Fig.2 plots outage probability with and without co-interferer when $\Omega_{S R}=\Omega_{R D}=1, \Omega_{I}=\Omega_{i}=0.1$, $\eta_{0}=\eta_{i}=\gamma_{0} / 2, C_{t h}=0.1 \mathrm{bits} / \mathrm{s} / \mathrm{Hz}$. It is clear that outage probability is degraded due to the presence of co-channel interference.

\section{Conclusions}

In this letter, a full-duplex relaying system with self-interference of the relay and co-channel interference of the destination is considered in detail, and then closed-form expressions for outage probability are provided to obtain the performance analysis and effect of interferences simply without statistical simulations.

\section{References}

[1] Laneman J., Tse D., and Wornell G.: 'Cooperative diversity in wireless networks: efficient protocols and outage behavior', IEEE Transactions on Information Theory, 2004, 50, (12), pp. 3062-3080

[2] Bletsas A., Khisti A., Reed D.P., A. Lippman A.: 'A simple cooperative diversity method based on network path selection', IEEE Journal on Selected Areas in Communications, 2006, 24, (3), pp. 659-672

[3] Suraweera A., Garg H.K. and Nallanathan A.: 'Performance analysis of two hop amplify-and-forward systems with interference at relay', IEEE Communication Letters, 2010, 14, (8), pp. 692-694

[4] Xu W., Zhang J. and Zhang P.: 'Outage probability of two-hop fixed-gain relay with interference at the relay and destination', IEEE Communications Letters, 2011, 15, (6), pp. 608-610

[5] Hemachandra K.T. and Beaulieu N.C.: 'Outage analysis of opportunistic scheduling in dual-hop multiuser relay network in the presence of interference', IEEE Transactions on Communications, 2013, 61, (5), pp. 1786-1796

[6] Ju H., Oh E., and Hong D.: ' Catching resource-devouring worms in next-generation wireless relay systems: two-way relay and full-duplex relay' , IEEE Communications Magazine, 2009, 47, (9), pp. 58-65

[7] Rui X.Y., Hou J. and Zhou L.L.: ' 'On the performance of full-duplex relaying with relay selection’ , Electronics Letters, 2010, 46, (25), pp. 1674-1676

[8] Wang T., Cano A., Giannakis G.B., Laneman J.N.: 'High-performance cooperative demodulation with decode-and-forward relays', IEEE Transactions on Communications, 2007, 55, (7), pp. 1427-1438 